\title{
Pregnancy and perinatal outcomes for women with Cystic Fibrosis: a UK population-based cross-sectional study, 2003-2017
}

\author{
Oluwaseun Esan ${ }^{1}$, Daniella Schlüter ${ }^{2}$, Rhiannon Phillips ${ }^{2}$, Rebecca Cosgriff ${ }^{2}$, Shantini \\ Parajothy $^{2}$, Deni Williams², Rachel Norman ${ }^{2}$, Siobhan Carr ${ }^{2}$, David Taylor Robinson ${ }^{1}$, and \\ Jamie Duckers ${ }^{3}$ \\ ${ }^{1}$ University of Liverpool \\ ${ }^{2}$ Affiliation not available \\ ${ }^{3}$ Cardiff and Vale University Health Board
}

May 24, 2021

\begin{abstract}
Objective To estimate the pregnancy rates and outcomes for women with cystic fibrosis (wwCF) in the UK compared to the general population and to explore the impact of the introduction of disease modifying treatments on pregnancy rates. Design A population-based cross-sectional study. Setting Electronic records of UK CF Registry Data ( $99 \%$ of all CF), and conceptions data for England and Wales (E\&W). Population All women aged 15-44 years who were pregnant between 2003-2017. Methods We calculated 3-yearly crude and age-specific pregnancy rates per 1,000 women years (wys), pregnancy rates for wwCF with a G551D mutation before and after Ivacaftor was introduced in 2012 and compared live birth rates. Main outcome measures Crude rates, age specific fertility, and maternal morbidity. Results The overall pregnancy rate was 23.5 (95\% CI 21.9-25.3) per 1,000 wys, $\sim 3.4$ fold difference to $\mathrm{E} \& \mathrm{~W}$ women (77.7). This pattern was evident in the age specific rates, except for those aged 40-44 years where the difference in rates was much less (wwCF 8.2 per 1,000 wys vs. 13.3 in E\&W). LB rate differences mirrored pregnancy rates (wwCF 17.4 per 1000 wys vs. $61.4 \mathrm{E} \& \mathrm{~W}$ women). Following the introduction of Ivacaftor, pregnancy rates in wwCF with G551D increased from 29.5 to 56.9 per 1000wys (2012-2014 to 2015-2017). Conclusions Pregnancy rates in wwCF are about a third of the rates in the general population but on the rise following the introduction of Ivacaftor. There is no indication that there is a reduced chance of a live birth in wwCF who become pregnant.
\end{abstract}

\section{Hosted file}

Esan et.al_Pregnancy outcomes_Manuscript_BJOG.docx available at https://authorea.com/users/ 362838/articles/523239-pregnancy-and-perinatal-outcomes-for-women-with-cystic-fibrosisa-uk-population-based-cross-sectional-study-2003-2017 